\title{
The Comparison of Area under the Curve of Serum Estradiol during Controlled Ovarian Stimulation between Long Gonadotrophin Releasing Hormone Agonist Protocol and Short Gonadotrophin Antagonist Protocol in Invitro Fertilization
}

\author{
Hartanto Bayuaji ${ }^{*}$, Wiryawan Permadi1 ${ }^{1}$ Herri S. Sastramihardja², \\ Heda Melinda Nazaruddin Nataprawira ${ }^{3}$
}

\begin{abstract}
${ }^{1}$ Department of Obstetrics \& Gynecology, Faculty of Medicine, Universitas Padjadjaran/Hasan Sadikin Hospital, West Java, Indonesia

${ }^{2}$ Department of Pharmacology and Therapy, Faculty of Medicine, Universitas Padjadjaran/Hasan Sadikin Hospital, West Java, Indonesia

${ }^{3}$ Department of Child Health, Faculty of Medicine, Universitas Padjadjaran/Hasan Sadikin Hospital, West Java, Indonesia

Email: ‘tantobayuaji@icloud.com,wiryawan_permadi@yahoo.com, herpst099@yahoo.com, heda_1155@yahoo.com
\end{abstract}

How to cite this paper: Bayuaji, H., Permadi, W., Sastramihardja, H.S. and Nataprawira, H.M.N. (2017) The Comparison of Area under the Curve of Serum Estradiol during Controlled Ovarian Stimulation between Long Gonadotrophin Releasing Hormone Agonist Protocol and Short Gonadotrophin Antagonist Protocol in Invitro Fertilization. Open Access Library Journal, 4: e3832.

https://doi.org/10.4236/oalib.1103832

Received: July 17, 2017

Accepted: August 4, 2017

Published: August 7, 2017

Copyright $\odot 2017$ by authors and Open Access Library Inc.

This work is licensed under the Creative Commons Attribution International License (CC BY 4.0).

http://creativecommons.org/licenses/by/4.0/

\begin{abstract}
Background: during stimulation, estradiol levels represent the dynamic hormonal interaction of follicle development. Some studies have been done to evaluate the influence of day-hCG estradiol assay and IVF outcome. However, this assay does not reflect the total exposure of estradiol throughout stimulation. Hence the concept of calculating the area under the curve (AUC) of estradiol during ovarian stimulation is thought to be a better parameter to evaluate total exposure of estradiol to the entire process of IVF. Methods: a retrospective analysis of data from a fertility center in a tertiary hospital. Between 2011-2016, there were long GnRH agonist cycles and short GnRH antagonist cycles eligible for analysis. Data including subject's characteristics, the level of estradiol on day-2, day-6, and day-hCG during controlled ovarian stimulation were obtained. Those data were plotted, and area under the curve of each subject was calculated. Statistical analysis was done using student t-test while $\mathrm{p}<0.05$ was considered significant. Results: there were no significant differences in subject's age ( 33.4 vs. 34.2 years, $\mathrm{p}=0.12$ ), day- 3 of menstrual cycle FSH level (7.1 vs. $7.3 \mathrm{mIU} / \mathrm{mL}, \mathrm{p}=0.64$ ), and day-3 estradiol level ( 35.1 vs. $36.5 \mathrm{pg} / \mathrm{mL}, \mathrm{p}=0.61$ ). The estradiol level on day-5 of COS was
\end{abstract}


$324.9(273.1) \mathrm{pg} / \mathrm{mL}$ and $363.3(293) \mathrm{pg} / \mathrm{mL}(\mathrm{p}=0.36)$, and at the day of hCG administration was $2461.8(2283.7) \mathrm{ng} / \mathrm{mL}$ and $2558.2(2043) \mathrm{ng} / \mathrm{mL}(\mathrm{p}=$ 0.76). Estradiol area under the curve for long protocol and short protocol was $9205.2(7683.7) \mathrm{pg} / \mathrm{mL}$ and $9732.9(6893.9) \mathrm{pg} / \mathrm{mL}$ respectively $(\mathrm{p}=0.63)$. Conclusion: our results indicate that there are no significant differences in the area under the curve of estradiol between two protocols.

\section{Subject Areas}

Gynecology \& Obstetrics

\section{Keywords}

Area under the Curve, Controlled Ovarian Stimulation, Estradiol, Long Agonist Protocol, Short Antagonist Protocol

\section{Introduction}

Controlled ovarian stimulation (COS) is an important and integral part of in vitro fertilization (IVF) program, with the goal to obtain more oocytes in supraphysiological conditions. This may lead to higher pregnancy chance, and the spare embryos can be cryo-stored for future transfer [1] [2]. The COS protocol mainly consists of exogenous follicle-stimulating hormone (FSH) administration using long-agonist protocol, and short-antagonist protocol. The long-agonist protocol induces hypophyseal desensitization to prevent premature luteinization, and significantly reduce cycle cancellation. The oocyte yield was considered to be higher compared to the short antagonist protocol. However, in a specific patient group such as polycystic ovary syndrome (PCOS), on the other hand, the short antagonist protocol completely blocks pituitary GnRH receptors and induces reversible and rapid gonadotrophin secretion. This may be achieved by administration of GnRH antagonist drugs such as cetrorelix and ganirelix [3] [4] [5].

The ovarian response during COS should be monitored adequately [2] [6] [7]. During COS, the ovarian produces E2 in supraphysiological level, and this may reflect ovarian response. The goal of COS monitoring was to ensure adequate ovarian response while avoiding complication potentials i.e., ovarian hyperstimulation syndrome or inadequate response due to inaccurate FSH dosage [2] [5] [8]. However, the serum E2 assay is mostly done by serial measurement during COS and may not reflect the actual exposure of E2. The area under the curve (AUC) concept, therefore proposed in several studies to calculate the amount of E2 exposure during COS [9] [10].

This study was aimed to compare the AUC during COS between long agonistand short antagonist protocol. We hypothesized that there was no significant difference in AUC between two protocols, which may reflect the same efficacy of both protocols. 


\section{Material and Methods}

This retrospective study was done by reviewing the records of female infertility patients in The Assisted Reproductive Technology Unit, Hasan Sadikin Hospital Bandung, Indonesia during the 2011-2016 period. Subjects included in this study were 20 - 40 years old infertile female who underwent IVF program. The inclusion criteria were a normal ovarian reserve (serum follicle stimulating hormone/FSH level $<10 \mathrm{mIU} / \mathrm{mL}$ and estradiol level $<70 \mathrm{pg} / \mathrm{mL}$ on day- 3 of the menstrual cycle, or anti-Mullerian hormone/AMH $>1.4 \mathrm{ng} / \mathrm{mL}$ ) [11], which was evaluated before the subject started the IVF program. Subjects with endometriosis, polycystic ovary syndrome, poor ovarian stimulation response, or ovarian hyperstimulation syndrome were excluded from this study. This study was approved by the Ethical Committee for Health Research, Hasan Sadikin Hospital Bandung. All patients have given their general consent before receiving management from the hospital.

The data obtained from medical records included subject's characteristics, serum estradiol level on the beginning of COS, day- 5 of COS, and at the day of hCG trigger administration during ovarian stimulation. Data then plotted in the curve with the $\mathrm{x}$-axis represents the day of menstrual cycle, and the $\mathrm{y}$-axis represents the estradiol level. The area under the curve was calculated by adding the area of rectangles and triangles for each time interval. The formula for calculating the AUC was proposed by Pruessner et al. as

$$
A U C=\frac{\{(m 2+m 1) t 1\}}{2}+\frac{\{(m 3+m 2) t 2\}}{2}
$$

where $m=$ measurement of estradiol level, and $t=$ time interval [12]. The mean of AUC of both groups was analyzed using student's t-test. Statistical analysis was performed using IBM SPSS version 24 (IBM Analytics, Chicago, IL, USA). The $\mathrm{p}$ value $<0.05$ was considered significant.

\section{Results}

During the study period, there were 185 subjects enrolled in the IVF program. There were 96 subjects using long agonist protocol and 89 subjects using short antagonist protocol. The characteristics of study subjects were presented in Table 1.

The serum E2 level during COS was presented in Table 2.

The AUC of E2 in long agonist protocol group was $9205.2(7683.7) \mathrm{pg} / \mathrm{mL}$, while in short antagonist protocol group was 9732.9 (6893.9) $\mathrm{pg} / \mathrm{mL}$. Using the student $\mathrm{t}$-test, there was no statistical difference of the AUC in both groups ( $\mathrm{p}=$ 0.63) (Figure 1).

\section{Discussion}

The age and ovarian reserve parameters were not significantly different between 
Table 1. Characteristics of the subjects.

\begin{tabular}{cccc}
\hline Characteristics & $\begin{array}{c}\text { Long agonist protocol } \\
\text { group } \mathrm{N}=96\end{array}$ & $\begin{array}{c}\text { Short antagonist protocol } \\
\text { group N = 89 }\end{array}$ & $\mathrm{P}^{*}$ \\
\hline Age, mean (SD) years & $33.3(3.4)$ & $34.2(4.2)$ & 0.12 \\
Day-2 FSH, mIU/mL & $7.1(1.9)$ & $7.3(4.7)$ & 0.64 \\
Day-2 E2, pg/mL & $35.1(18.6)$ & $36.5(18.0)$ & 0.61 \\
\hline
\end{tabular}

*student t-test.

Table 2. The comparison of E2 level.

\begin{tabular}{cccc}
\hline & $\begin{array}{c}\text { Long agonist } \\
\text { protocol group } \mathrm{N}=96\end{array}$ & $\begin{array}{c}\text { Short antagonist } \\
\text { protocol group N = 89 }\end{array}$ & $\mathrm{P}^{*}$ \\
\hline E2 on the beginning of COS & $35.1(18.6)$ & $36.5(18.0)$ & 0.61 \\
E2 level on day-5 of COS & $324.9(273.1)$ & $363.3(293)$ & 0.36 \\
E2 level at the day of hCG administration & $2461.8(2283.7)$ & $2558.2(2043)$ & 0.76 \\
\hline
\end{tabular}

${ }^{*}$ student t-test.

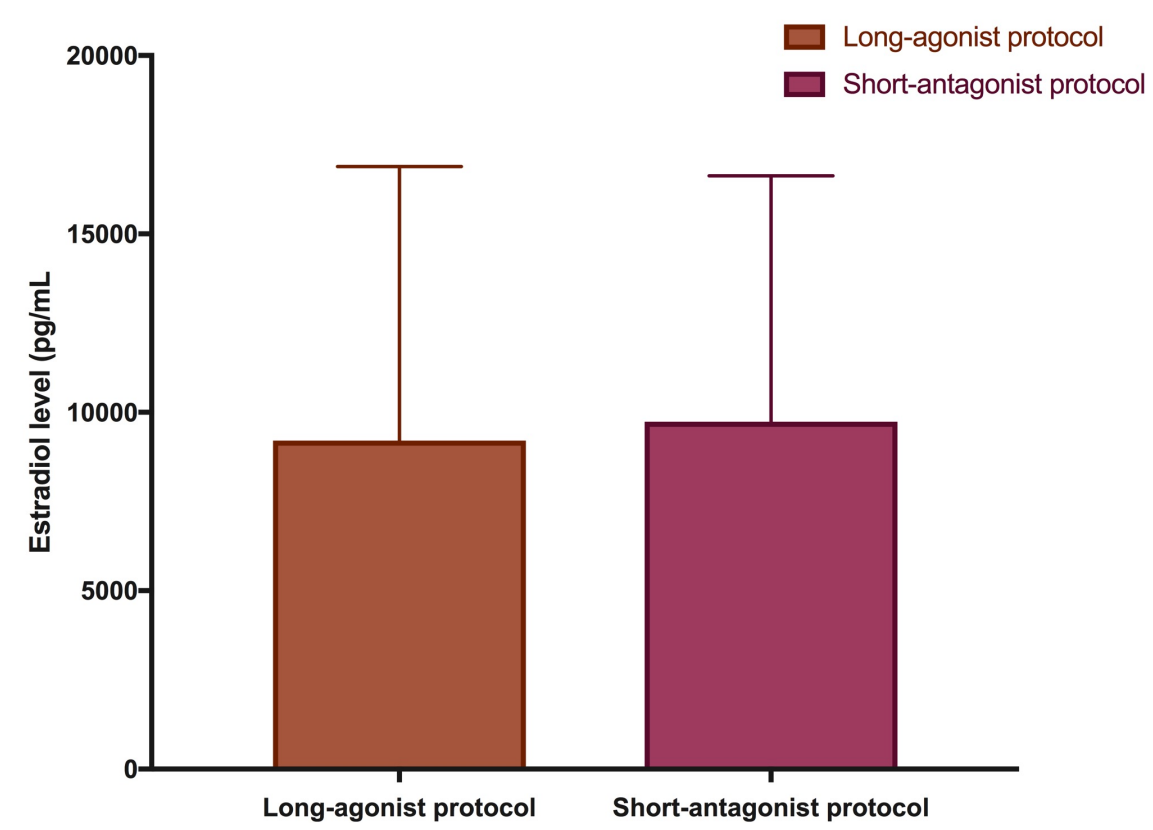

Figure 1. The comparison of area under the curve of serum estradiol from both study groups.

both study groups, reflecting the similar characteristics. Long agonist protocol was related to more severe suppression of pituitary, resulting downregulated condition before ovarian stimulation commenced. Therefore, this protocol may not be suitable for patients with diminished ovarian reserve or advanced age. Our subjects' characteristics in long agonist group the mean of age were still below 35 years old, the age of decreased ovarian reserve would be anticipated. The day-3 FSH and E2 level, which used for evaluating the ovarian reserve, did not significantly differ as well. Due to its simplicity and convenience, the short antagonist protocol now became much applied in our hospital. However, the ques- 
tions may arise from the patients whether the short antagonist protocol may yield the same results as the already established long agonist protocol. To answer the question, we evaluate the dynamics of serum E2 level, the marker that may reflect the ovarian response during COS. There was no significant difference of E2 level throughout COS. After calculating the AUC of E2, there was no significant difference of AUC in both groups. This may reflect the similar efficacy of both protocols.

The E2 dynamics during COS was evaluated during COS for several reasons: to ensure the adequate ovarian response which may yield the optimum results, and to prevent the probability of the complication such as ovarian hyperstimulation syndrome [13]. The final response of COS usually evaluated by measuring E2 level before the administration of hCG which served as a trigger to induce oocyte maturation. However, this might not reflect the total exposure, or total estradiol secreted by the developing follicles [9]. Hence the concept of area under the curve calculation was then introduced. This was done by calculating the sum of the area in estradiol assay result in day to day basis. In our hospital, the serum estradiol assay was done in the commencement, on the day- 5 of stimulation, and at the end of stimulation. This might lead to the limited data obtained and hence became the limitation of our study. Mitwally et al. conducted the study using the graph plotted based on the estradiol level on the day-to-day basis in long agonist protocol [9]. The AUC of serum E2 was 9357 (4555) pg/mL. They conclude that AUC of estradiol reflected the amount of E2 produced during COS more accurately. Kutlu et al. conducted a study to evaluate whether the AUC of E2 might be used as a predictor of clinical pregnancy or implantation failure. They found that there was a significant difference between AUC of E2 in women with successful implantation and those whose implantation was failed [10]. They concluded that the AUC of E2 might be used for predicting implantation success.

The serum E2 resulted from COS was considered in supraphysiologic level, that in several circumstances may interfere the receptivity of endometrium, leading to implantation failure. This was observed in several studies, who found the lower implantation rate in high estradiol level condition during IVF ET cycle [10] [14] [15]. However, the difference in pregnancy results was not the primary goal of this study.

The limitation of this study was a relatively small subject to analyze and less serum estradiol assay checkpoint compared with other studies. The day-to-day estradiol assay will produce smoother curve, therefore the area under the curve calculation will reveal more accurate results. However, our results was in similar range with other study [9].

\section{Conclusion}

There was no significant difference of area under the curve of serum estradiol between long agonist protocol and short antagonist protocol in controlled ova- 
rian stimulation.

\section{References}

[1] Farquhar, C., Marjoribanks, J., Brown, J., Fauser, B., Lethaby, A., Mourad, S., et al. (2017) Management of Ovarian Stimulation for IVF: Narrative Review of Evidence Provided for World Health Organization Guidance. Reproductive Biomedicine Online, 35, 3-16. https://doi.org/10.1016/j.rbmo.2017.03.024

[2] Kwan, I., Bhattacharya, S., Kang, A. and Woolner, A. (2014) Monitoring of Stimulated Cycles in Assisted Reproduction (IVF and ICSI). Cochrane Database of Systematic Reviews, 8, Article ID: CD005289. https://doi.org/10.1002/14651858.CD005289.pub3

[3] Macklon, N.S., Stouffer, R.L., Giudice, L.C. and Fauser, B.C. (2006) The Science behind 25 Years of Ovarian Stimulation for in Vitro Fertilization. Endocrine Reviews, 27, 170-207. https://doi.org/10.1210/er.2005-0015

[4] Santos, M.A., Kuijk, E.W. and Macklon, N.S. (2010) The Impact of Ovarian Stimulation for IVF on the Developing Embryo. Reproduction, 139, 23-34.

https://doi.org/10.1530/REP-09-0187

[5] Vandekerckhove, F., Gerris, J., Vansteelandt, S. and De Sutter, P. (2014) Adding Serum Estradiol Measurements to Ultrasound Monitoring Does Not Change the Yield of Mature Oocytes in IVF/ICSI. Gynecological Endocrinology, 30, 649-652. https://doi.org/10.3109/09513590.2014.912267

[6] Hendriks, D.J., Klinkert, E.R., Bancsi, L.F., Looman, C.W., Habbema, J.D., Velde, E.R., et al. (2004) Use of Stimulated Serum Estradiol Measurements for the Prediction of Hyperresponse to Ovarian Stimulation in in Vitro Fertilization (IVF). Journal of Assisted Reproduction and Genetics, 21, 65-72. https://doi.org/10.1023/B:JARG.0000027016.65749.ad

[7] Macklon, N.S. and Fauser, B.C. (2005) Gonadotrophins in Ovulation Induction. Reproductive Biomedicine Online, 10, 25-31. https://doi.org/10.1016/S1472-6483(11)60387-8

[8] Martins, W.P., Vieira, C.V., Teixeira, D.M., Barbosa, M.A., Dassuncao, L.A. and Nastri, C.O. (2014) Ultrasound for Monitoring Controlled Ovarian Stimulation: A Systematic Review and Meta-Analysis of Randomized Controlled Trials. Ultrasound in Obstetrics Gynecology, 43, 25-33. https://doi.org/10.1002/uog.12566

[9] Mitwally, M.F., Bhakoo, H.S., Crickard, K., Sullivan, M.W., Batt, R.E. and Yehl, J. (2005) Area under the Curve for Estradiol Levels Do Not Consistently Reflect Estradiol Levels on the Day of HCG Administration in Patients Undergoing Controlled Ovarian Hyperstimulation for IVF-ET. Journal of Assisted Reproduction and Genetics, 22, 57-63. https://doi.org/10.1007/s10815-005-1494-4

[10] Kutlu, T., Ozkaya, E., Ayvaci, H., Devranoglu, B., Sanverdi, I., Sahin, Y., et al. (2016) Area under Curve of Temporal Estradiol Measurements for Prediction of the Detrimental Effect of Estrogen Exposure on Implantation. International Journal of $G y$ naecology and Obstetrics, 135, 168-171. https://doi.org/10.1016/j.ijgo.2016.04.015

[11] Homburg, R. (2014) Ovulation Induction and Controlled Ovarian Stimulation. Springer International Publishing, Gewerbestrasse.

[12] Pruessner, J.C., Kirschbaum, C., Meinlschmid, G. and Hellhammer, D.H. (2003) Two Formulas for Computation of the Area under the Curve Represent Measures of Total Hormone Concentration versus Time-Dependent Change. Psychoneuroendocrinology, 28, 916-931. https://doi.org/10.1016/S0306-4530(02)00108-7

[13] Kwan, I., Bhattacharya, S., McNeil, A. and Van Rumste, M.M. (2008) Monitoring of 
Stimulated Cycles in Assisted Reproduction (IVF and ICSI). Cochrane Database of Systematic Reviews, 2, Article ID: CD005289.

https://doi.org/10.1002/14651858.CD005289.pub2

[14] Valbuena, D., Martin, J., de Pablo, J.L., Remohi, J., Pellicer, A. and Simon, C. (2001) Increasing Levels of Estradiol Are Deleterious to Embryonic Implantation because They Directly Affect the Embryo. Fertility and Sterility, 76, 962-968. https://doi.org/10.1016/S0015-0282(01)02018-0

[15] Blumenfeld, Z. (2015) Why More Is Less and Less Is More When It Comes to Ovarian Stimulation. Journal of Assisted Reproduction and Genetics, 32, 1713 1719. https://doi.org/10.1007/s10815-015-0599-7

\section{List of Abbreviations}

AMH: anti-Müllerian hormone;

AUC: area under the curve;

COS: controlled ovarian stimulation;

E2: estradiol;

ET: embryo transfer;

FSH: follicle-stimulating hormone;

$\mathrm{GnRH}$ : gonadotrophin-releasing hormone;

IVF: in vitro fertilization;

PCOS: polycystic ovary syndrome.

Submit or recommend next manuscript to OALib Journal and we will provide best service for you:

- Publication frequency: Monthly

- 9 subject areas of science, technology and medicine

- Fair and rigorous peer-review system

- Fast publication process

- Article promotion in various social networking sites (LinkedIn, Facebook, Twitter, etc.)

- Maximum dissemination of your research work

Submit Your Paper Online: Click Here to Submit

Or Contact service@oalib.com 